\title{
Qualité physico-chimique des eaux usées de la station d'épuration de la ville de S'anaa (Yémen)
}

\author{
Shaker RAWEH ${ }^{1}$, Driss BELGHYTI ${ }^{1 *}$, Abdel Baki AL-ZAEMEY ${ }^{2}$ \\ Youssef EL GUAMRI ${ }^{1}$ et Khadija ELKHARRIM ${ }^{1}$ \\ ${ }^{I}$ Laboratoire de Biologie et Santé, Équipe de Recherche en Environnement et Parasitologie / UFR Doctorale \\ "Parasitologie Comparée : Applications Médicales et Vétérinaires », Faculté des Sciences, Université Ibn \\ Tofaïl, BP 133, code postale : 14000 Kénitra, Maroc.E-mail : shaker.raweh@hotmail.com \\ ${ }^{2}$ Faculté d'Agriculture, Université S'anaa, République du Yémen. E-mail : alzaemey@yahoo.com \\ *Auteur correspondant ; E-mail : belghyti@hotmail.com
}

\section{RESUME}

Dans le souci de réduire le flux des polluants présents dans les eaux usées des agglomérations urbaines de la république de Yémen, une étude préliminaire a été entreprise au niveau de la station d'épuration de la ville de S'ANAA (Yémen). Cette étude avait comme premier objectif d'estimer la pollution physico-chimique globale dans les effluents de la STEP. Il s'agissait d'abord, d'identifier les sources et les origines de pollution à l'entrée de la station, et ensuite d'étudier les performances épuratoires de la station d'épuration. Le but est de ramener les teneurs des éléments polluants dans l'eau en dessous des valeurs fixées par les normes internationales. Des mesures d'indicateurs de qualité ont été effectuées pendant six mois sur des échantillons prélevés suivant une fréquence de quatre fois par mois. Les rendements épuratoires moyens atteignent 90,99\% en DBO5, 89,99\% en DCO et 86,4\% en MES. L'élimination des orthophosphates est assez élevée avec un rendement moyen de 68\%. De manière globale, les teneurs moyennes en sels restent assez élevées par rapport à la limite tolérable pour un rejet de raffinerie des eaux usées dans un milieu récepteur. Par ailleurs, on a révélé une moyenne de $100,5 \mathrm{mg} / \mathrm{l}( \pm 12,02)$ pour les NH4 avec un taux d'abattement de $33 \%$ et une moyenne de $2152,75 \mu \mathrm{s} / \mathrm{cm}( \pm 151,49)$ pour les conductivités électriques.

(C) 2011 International Formulae Group. All rights reserved.

Mots clés : Eaux usées, traitement, performances épuratoires, S'anaa, Yémen.

\section{INTRODUCTION}

Actuellement, le monde souffre de la pollution environnementale. Cette pollution est due généralement à l'augmentation des activités humaines dans touts les secteurs et elle cause des dégâts pour la santé humaine et altère l'équilibre écologique. Pour cette raison, tous les pays ont déployé des efforts pour résoudre cette problématique surtout des raretés et l'altération des qualités des eaux à cause de la diversité des sources de la pollution hydrique. La difficulté de gestion de cette pollution par les pays en voie de développement nécessite des projets avec des grands budgets pour résoudre ce problème. La plupart de ces pays ont installé des stations de traitement des eaux usées mais la gestion de ces stations est différente selon le type d'activités de chaque pays. Pour la république du Yémen, pays qui souffre de la 
rareté de l'eau avec un taux de consommation global de l'eau estimé à $3400 \mathrm{M} \mathrm{m}^{3}$ en 2000 , les ressources renouvelables sont de l'ordre de $2500 \mathrm{M} \mathrm{m}^{3}$ (1000 $\mathrm{M} \mathrm{m}^{3}$ sous forme des eaux superficielles et $1500 \mathrm{M} \mathrm{m}^{3}$ sous forme des eaux souterraines). On remarque ici un déficit hydrique estimé en $900 \mathrm{M} \mathrm{m}^{3}$ exploité à partir des nappes phréatiques (MPCI, 2005).

La moyenne des précipitations annuelles au Yémen varie entre 50 et 100 $\mathrm{mm}$, en minimum, et $800 \mathrm{~mm}$ en maximum, d'où une faible moyenne individuelle annuelle par habitant dans ce pays de l'ordre de $125 \mathrm{~m}^{3}$ seulement (NWSSIP, 2005).

Le Ministère des Eaux et de l'Environnement (Yémen) est préoccupée par les problèmes de la pollution des eaux usées. C'est dans cette optique, que la Direction Générale de l'Eau et d'Assainissement (S'ANAA) se veut être conforme aux normes de rejets des eaux usées. Elle a installé une STEP par boues activées. Le résultat le plus remarquable de cette étude a été évidemment la mise en application de mesures de lutte contre la pollution, l'adoption d'un plan de surveillance générale le long de la chaîne d'épuration et d'un plan d'intervention contre la pollution par les eaux usées brutes et épurées.

\section{MATERIEL ET METHODES}

Au Yémen, certaines agglomérations urbaines ont bénéficié d'un réseau d'assainissement liquide et l'installation des stations de traitement des eaux usées surtout dans les principales villes. La station de traitement des eaux usées de la ville de S'anaa (Yémen), notre zone d'étude, est l'une des principales stations du pays où l'effectif des habitants est très élevé à cause de l'extension urbaine et démographique. Cette station a été créée pour traiter et réutiliser les eaux usées pour irriguer les espaces verts. La date de lancement de cette station est le 22/05/2000, cette station est dirigée par la Direction Générale des Eaux et
d'Assainissement du Ministère de l'Eau et de l'Environnement (Figure 1).

La source principale de l'eau pour les habitants c'est les puits dans la plupart des villes Yéménite et les zones rurales avec l'existence des barrages, mais c'est rare que les eaux de ces barrages soient utilisées pour la consommation.

\section{Station de traitement des eaux usées de la ville de S'anaa (Yémen)}

La station est située au Nord Est de l'aéroport S'anaa international de la province de Bani Alharit (sens d'écoulement de l'oued Al Kharid), sur une hauteur de $2200 \mathrm{~m}$ de la mer. Actuellement, les eaux usées de la station d'épuration de la ville de S'anaa sont traitées dans la perspective de les réutiliser pour l'irrigation des champs agricoles selon les normes internationales. La capacité de traitement de cette station en eaux usées qui est de l'ordre de $50.000 \mathrm{~m}^{3}$ a atteint actuellement $43.000 \mathrm{~m}^{3}$ (IEA, 2006).

\section{Diagnostic environnemental de la division de Bani Alharit (zone d'étude - station de traitement des eaux usées de Sanaa) \\ La STEP située dans la zone de Bani} Alharit, et dans la $7^{\text {ème }}$ zone climatique qui s'étend sur les zones Est et le Nord des séries montagneuses de la ville de Rdaa jusqu'à la ville de Saada en Nord. Cette zone couvre presque $500 \mathrm{~m}^{2}$ (MPCI, 2005).

Les eaux usées brutes de certains quartiers ne sont pas raccordées aux réseaux d'assainissement liquide de la ville de S'anaa. Les eaux usées d'abattoir sont transportées par des véhicules citernes à la station de traitement. Le contrôle de ces véhicules citernes est obligatoire pour éviter l'entrée des déchets solides à la station. Quand les eaux usées sont chargées par ces déchets solides, elles sont évacuées directement dans l'oued sans traitement, parfais, on remarque le changement de la couleur des eaux de l'oued (rouge) à cause des effluents de l'abattoir. Des risques sanitaires sont 
éventuellement présents surtout que les eaux de l'oued sont réutilisées pour l'irrigation.

\section{Procédé de traitement (Fonctionnement de la station de traitement)}

La STEP a été installée par la société Anglaise Horad Hemvri pour servir presque 1500000 habitants avec une capacité maximale de $50000 \mathrm{~m}^{3} / \mathrm{J}$ (Figure 2). La date de lancement de fonctionnement de la station est le 22 Mai 2000. Les eaux usées brutes à l'entrée de la STEP doivent présenter les caractéristiques physico-chimiques suivantes:

Eaux usées brutes (Entré): DBO5 = $500 \mathrm{mg} / \mathrm{l}, \mathrm{MES}=750 \mathrm{mg} / \mathrm{l}, \mathrm{NH} 4=100 \mathrm{mg} / \mathrm{l}$.

Eaux usées traitées (Sortie): DBO5 = $30 \mathrm{mg} / \mathrm{l}, \mathrm{MES}=30 \mathrm{mg} / \mathrm{l}$.

La STEP permet de traiter des eaux usées brutes de la ville de S'anaa très chargées en matières organiques. Elle comporte un poste de relevage (puisard), il s'agit d'un bassin en amont du traitement physico-chimique d'une capacité de 50000 $\mathrm{m}^{3} / \mathrm{J}$ et un débit de $2300 \mathrm{~m}^{3} / \mathrm{h}$. Ce bassin a pour rôle d'absorber toutes les fluctuations et d'assurer l'alimentation continue des ouvrages de traitement dans les conditions optimales. Il permet aussi un prétraitement par déshuilages et dégraissages avant d'alimenter les Bassins d'aération de la STEP. Ces bassins d'aération ont pour objectif la dégradation de la matière organique. En suite, les eaux décantées sont traitées par des procédés physico-chimiques qui consistent à éliminer la pollution colloïdale telle que les matières en suspension fine (limons, produits de corrosion). Cette épuration est réalisée par chloration. En suite, ces effluents sont traités par voie biologique (Boues activées), afin d'éliminer la pollution dissoute et biodégradable. Avant le déversement dans le milieu récepteur (Oued Al Kharid), les eaux usées traitées sont acheminées vers le bassin terminal pour satisfaire aux normes de rejets. Les boues sont épaissies, déshydratées et réutilisées comme des engrais dans l'agriculture (Figure 2).

Les eaux de pluies et de ruissellement au niveau de la STEP sont collectées dans un bassin d'orage.

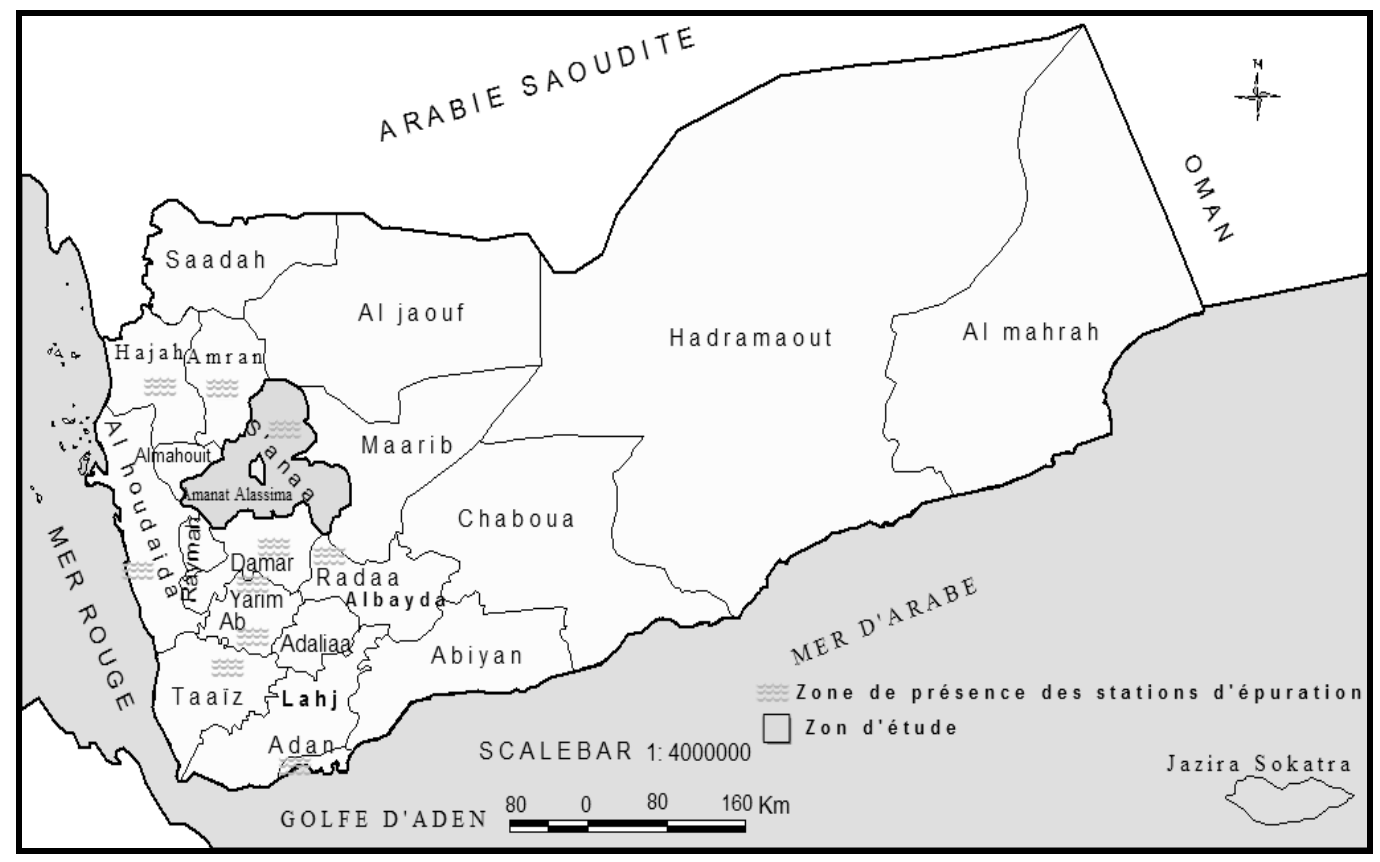

Figure 1: Localisation de la zone d'étude (ville de S'anaa, Yémen). 


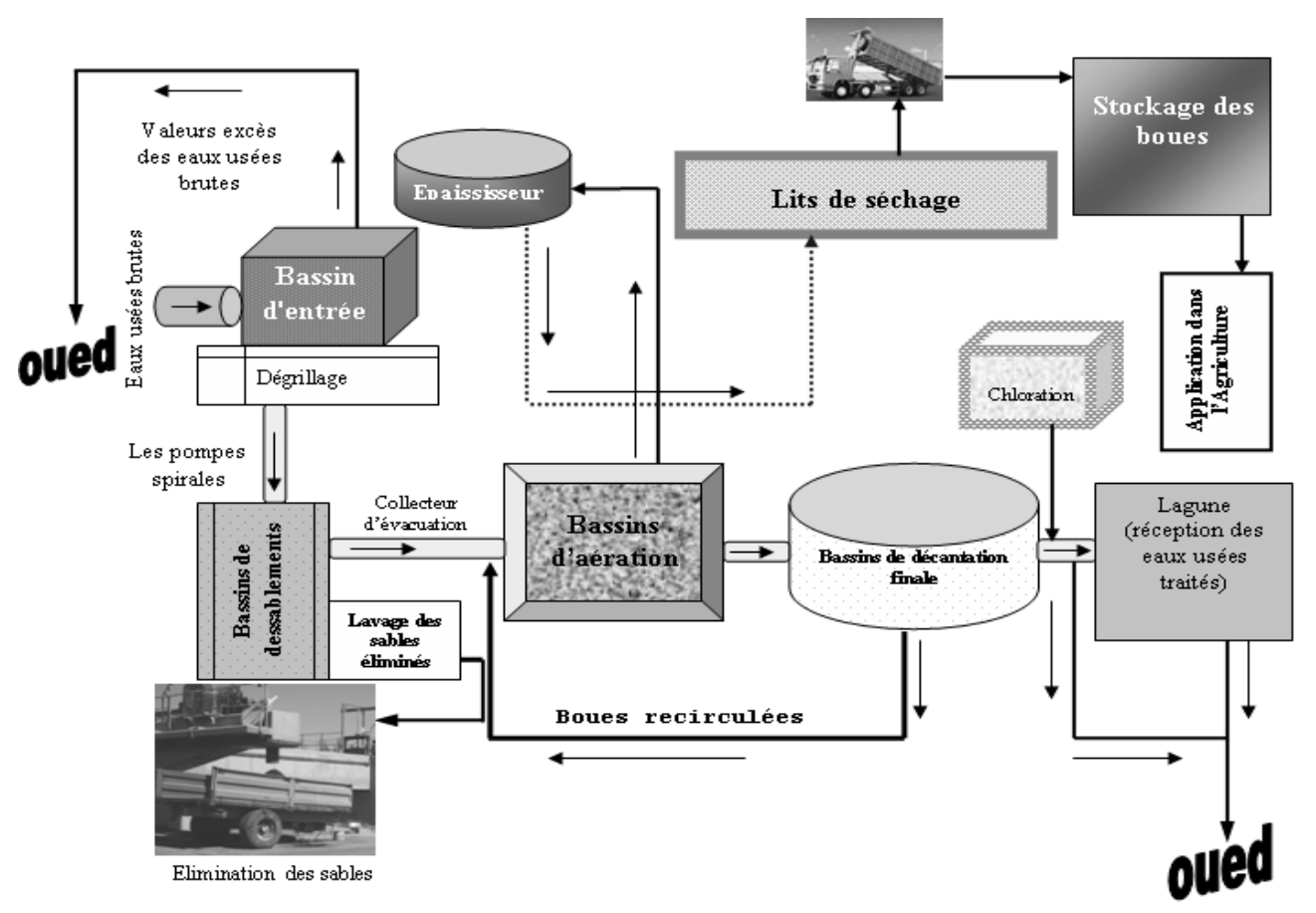

Figure 2: Schéma de la station de traitement des eaux usées de la ville de S'anaa (Yémen).

\section{Méthodologie d'échantillonnage et de mesure \\ L'échantillonnage a été effectué quatre} fois par mois sur une période de 6 mois (de Janvier à juin 2007) à l'entrée et à la sortie du décanteur final afin de suivre les performances épuratoires de la STEP. Les paramètres physico-chimiques globaux (conductivité électrique, $\mathrm{pH}$ ) ont été mesurés in situ au moyen d'un "Conductivity Meter" et "pH-meters" (Référence: sensION7 Benchtop Conductivity Meter, with electrode, 1 -meter cable, $115 \mathrm{~V}$ et HANNA INSTRUMENTS, HI 9622, microcomputer pH-meters) sur des échantillons composites. La DBO5, la DCO et les MES ont été mesurés sur des échantillons composites constitués en proportions égales d'échantillons ponctuels prélevés à l'entrée et à la sortie de la STEP. Pour les matières en suspension (MES), la filtration est réalisée sur membrane poreuse de $0,45 \mu \mathrm{m}$, puis le séchage à $105^{\circ} \mathrm{C}$. La demande chimique en oxygène est analysée par oxydation en excès au dichromate de potassium à chaud en milieu acide (méthode AFNOR). La DBO5 a été évaluée par la méthode manométrique basée sur le principe WARBURG (AFNOR, T90-103).

\section{RESULTATS}

Caractéristiques de l'effluent brut (à l'entrée de la STEP)

Les caractéristiques de l'effluent brut admis à l'entrée de la STEP sont présentées dans le Tableau 1.

\section{Rendements épuratoires}

Les rendements attendus du système de traitement des eaux usées par boues activées sont calculés à la base des moyennes mensuelles de chaque paramètre. Les concentrations moyennes de l'effluent à l'entrée et à la sortie de la STEP pour différents paramètres sont résumées dans le Tableau 3 et dans les Figures 3, 4 et 5.

\section{Caractéristiques de l'effluent traité (à la sortie de la STEP)}

Les caractéristiques de l'effluent traité à la sortie de la STEP sont présentées dans le Tableau 2. 
Tableau 1 : Composition physico-chimique des eaux usées brutes à l'entrée de la STEP.

\begin{tabular}{|c|c|c|c|c|c|c|}
\hline Paramètres & $\begin{array}{c}\text { Valeur } \\
\text { Min. }\end{array}$ & Moyenne & $\begin{array}{c}\text { Valeur } \\
\text { Max. }\end{array}$ & $\begin{array}{c}\text { Écart- } \\
\text { type }\end{array}$ & $\begin{array}{c}\text { Nombre } \\
\text { d'échantillon }\end{array}$ & $\begin{array}{c}\text { Normes } \\
\text { Yéménites }\end{array}$ \\
\hline pH & 7,21 & 7,43 & 7,55 & 0,117 & 24 & $\begin{array}{c}5,5-9,5^{*} \\
6,5-8,4 * * *\end{array}$ \\
\hline $\begin{array}{l}\text { DBO5 } \\
(\mathrm{mg} / \mathrm{L})\end{array}$ & 1083,5 & 1137 & 1199 & 49,76 & 24 & $\begin{array}{c}800 * \\
150 * *\end{array}$ \\
\hline DCO (mg/L) & 1784 & 1888,53 & 1960 & 87,89 & 24 & $\begin{array}{l}2100 * \\
500 * *\end{array}$ \\
\hline $\mathrm{NH}_{4}{ }^{+}(\mathrm{mg} / \mathrm{L})$ & 131,93 & 149,28 & 166,63 & 24,54 & 24 & 5- $30 * * *$ \\
\hline $\mathrm{PO}_{4}(\mathrm{mg} / \mathrm{L})$ & 97,77 & 107,307 & 112,77 & 5,83 & 24 & $\begin{array}{c}50 * \\
30 * *\end{array}$ \\
\hline $\begin{array}{l}\text { Conductivité } \\
(\mu \mathrm{s} / \mathrm{cm})\end{array}$ & 1838 & 2028,3 & 2198,75 & 136,54 & 24 & $700-4000 * * *$ \\
\hline M.E.S & 680 & 813,5 & 885 & 89,14 & 24 & $1100 *$ \\
\hline
\end{tabular}

Tableau 2 : Composition physico-chimique des eaux usées traitées à la sortie de la STEP.

\begin{tabular}{|c|c|c|c|c|c|c|}
\hline Paramètres & $\begin{array}{l}\text { Valeur } \\
\text { Min. }\end{array}$ & Moyenne & $\begin{array}{l}\text { Valeur } \\
\text { Max. }\end{array}$ & Écart-type & $\begin{array}{c}\text { Nombre } \\
\text { d'échantillon }\end{array}$ & $\begin{array}{c}\text { Normes } \\
\text { Yéménites }\end{array}$ \\
\hline pH & 7,5 & 7,7 & 7,8 & 0,102 & 24 & $\begin{array}{c}5,5-9,5^{*} \\
6,5-8,4^{* * *}\end{array}$ \\
\hline DBO5 (mg/L) & 91 & 102,416 & 111,75 & 8,197 & 24 & $\begin{array}{c}800 * \\
150 * *\end{array}$ \\
\hline DCO (mg/L) & 154,67 & 189,04 & 203,33 & 20,27 & 24 & $\begin{array}{l}2100 * \\
500 * *\end{array}$ \\
\hline $\mathrm{NH}_{4}^{+}(\mathrm{mg} / \mathrm{L})$ & 92 & 100,5 & 109 & 12,02 & 24 & 5- $30 * * *$ \\
\hline $\mathrm{PO}_{4}(\mathrm{mg} / \mathrm{L})$ & 26,68 & 34,60 & 42,73 & 5,54 & 24 & $\begin{array}{c}50 * \\
30 * *\end{array}$ \\
\hline $\mathrm{NO}_{3}(\mathrm{mg} / \mathrm{L})$ & 12,73 & 14,84 & 17,73 & 1,947 & 24 & $45 * * * *$ \\
\hline $\begin{array}{l}\text { Conductivité } \\
(\mu \mathrm{s} / \mathrm{cm})\end{array}$ & 1970,5 & 2152,75 & 2399 & 151,49 & 24 & 700- $4000 * * *$ \\
\hline M.E.S & 81,43 & 110,6 & 142,29 & 25,79 & 24 & $1100 *$ \\
\hline
\end{tabular}


Tableau 3 : Rendement de la chaîne de traitement des indices de pollution.

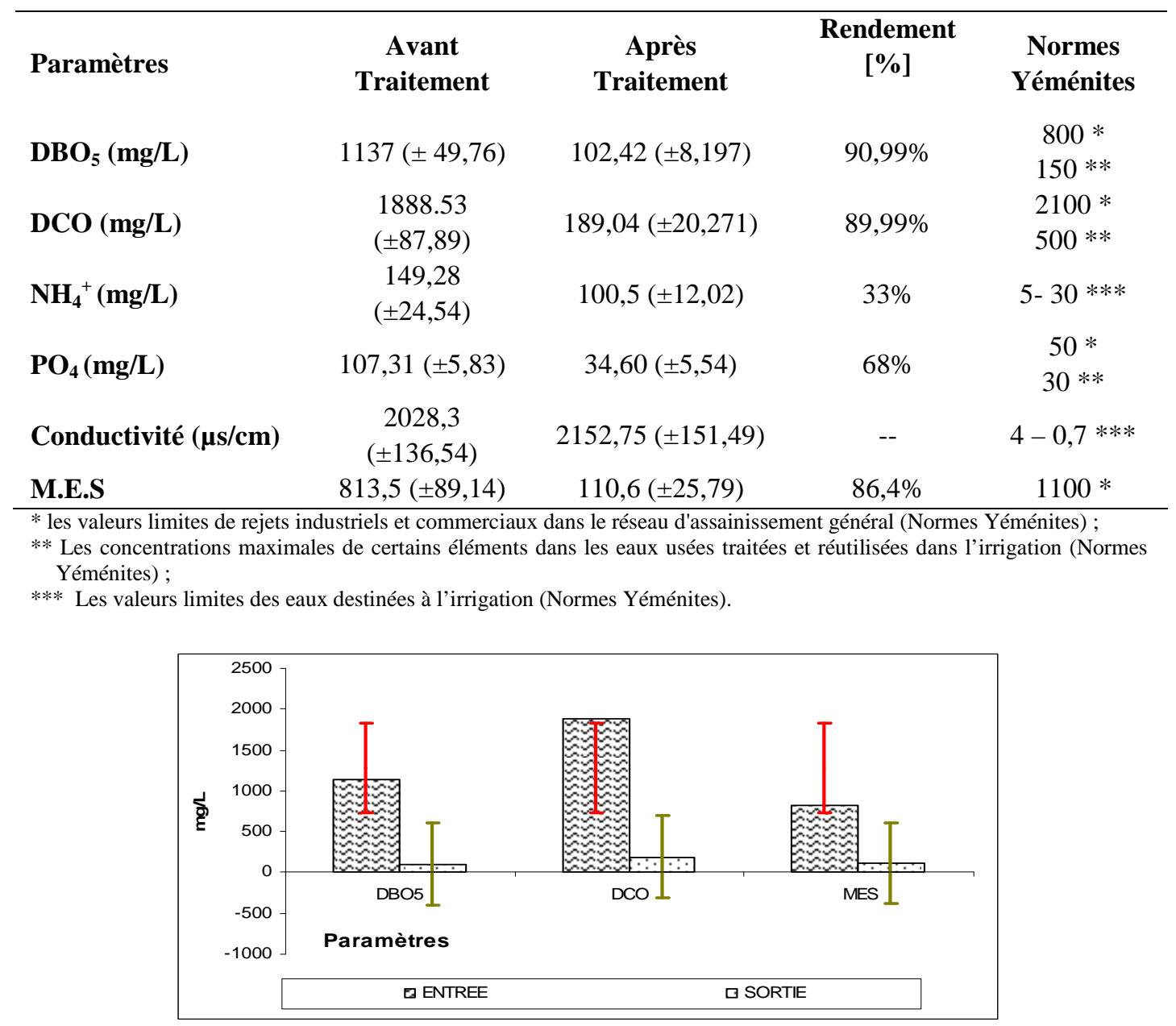

Figure 3 : Variation des concentrations moyennes mensuelles en $\mathrm{DBO}_{5}, \mathrm{DCO}$ et $\mathrm{MES}(\mathrm{mg} / \mathrm{l}) \mathrm{de}$ l'effluent brut et l'effluent traité.

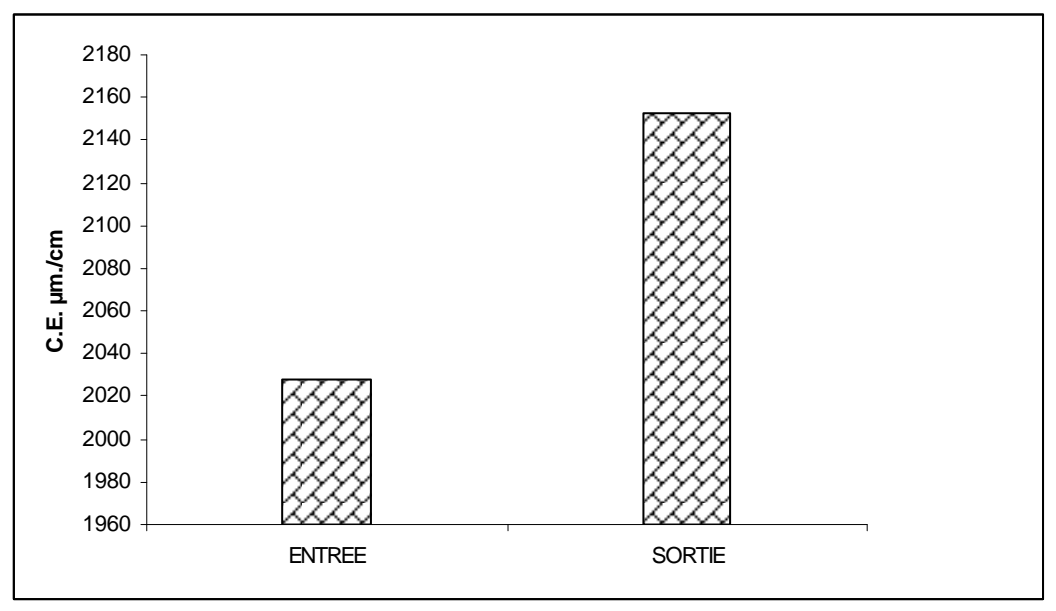

Figure 4: Variation des concentrations moyennes mensuelles de la Conductivité électrique $(\mu \mathrm{s} / \mathrm{cm})$ de l'effluent brut et l'effluent traité. 


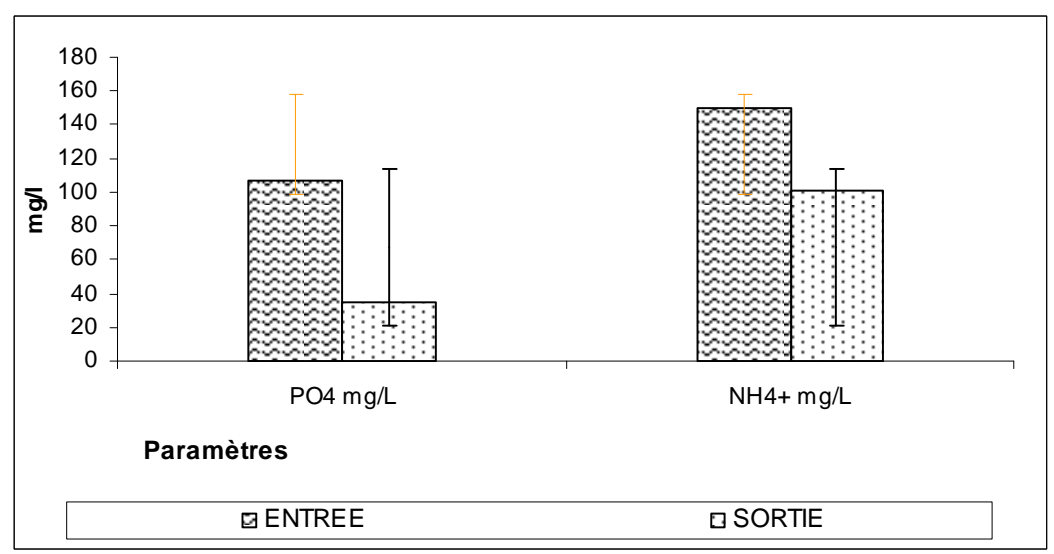

Figure 5 : Variation des concentrations moyennes mensuelles en $\mathrm{PO}_{4}^{--}$et $\mathrm{NH}_{4}{ }^{+}(\mathrm{mg} / \mathrm{l})$ de l'effluent brut et l'effluent traité.

\section{DISCUSSION}

Les valeurs moyennes du $\mathrm{pH}$ à l'entrée de la station de traitement des eaux usées oscillent entre 7,2 et 7,5 comme valeurs extrêmes minimales et maximales avec une moyenne de 7,4 , ce qui est de nature à favoriser le développement bactérien nécessaire à la dégradation biologique des polluants organiques (Maiga et al., 2006). La plupart des organismes ne peuvent pas tolérer un pH supérieur à 9,5 ou inférieur à 4 .

On recommande généralement un $\mathrm{pH}$ entre 6,5 et 7,5 pour les unités de traitement des eaux usées (Metcalf et Eddy, 1991), car un faible $\mathrm{pH}$ favorise la croissance de champignons filamenteux et d'autres organismes responsables des boues flottantes (Arcand et al., 1989). La DBO5 évoluent dans une gamme de valeurs situées entre 1083,5 à $1199 \mathrm{mg} / \mathrm{l}$ avec une valeur moyenne de $1137 \mathrm{mg} / \mathrm{l}$. Les valeurs de la DBO5 trouvées sont nettement inférieures à celles obtenues par RAWEH (2005), EL GUAMRI et BELGHYTI (2006).

Les teneurs en MES indiquent que l'effluent contient une quantité importante de particules insolubles et décantables. Les MES ont des concentrations comprises entre 680 et $885 \mathrm{mg} / \mathrm{l}$, avec une moyenne de 813,5 mg/l. Les valeurs de la DCO et de la DBO5 restent dans la gamme des valeurs de référence pour les eaux usées domestiques avec un rapport de $\mathrm{DCO} / \mathrm{DBO} 5 \leq 2$. Ce rapport est légèrement supérieur à 1,66 , ce qui caractérise un effluent un peu biodégradable (Metcalf et Eddy, 1991). On est dans une situation de forte variabilité de la qualité de l'effluent admis à l'entrée de la station de traitement des eaux usées de la STEP de la ville de S'anaa.

$\mathrm{Au}$ terme des résultats obtenus, les effluents bruts à l'entrée de la STEP, ne répondent pas aux normes de rejets directs d'une STEP, en DBO5, $\mathrm{NH}_{4}{ }^{+}$et $\mathrm{PO}_{4}{ }^{3-}$. Tandis que, le $\mathrm{pH}$, et les MES, la DCO et la conductivité électrice totaux mesurés sont dans les limites de ces rejets fixés par les autorités Yéménites (ANRE, 1999a; ANRE, 1999b).

Le rapport DBO5/DCO est de l'ordre de 0,6. Ce résultat permettrait de prédire que les eaux usées brutes de la STEP sont polluées par une pollution inorganique assez forte. Par ailleurs, le rapport DCO/DBO5 est de l'ordre de 1,66, valeur assez élevée, ce qui indique que la Matière Oxydable, estimée en 1387,51 mg/l dans les eaux usées brutes de la STEP, est difficilement dégradable. Ce résultat pourrait être attribué aux rejets des industries de textile, pharmaceutiques, agroalimentaires, stations de lavage des voitures, raccordées à la STEP.

Les résultats sont similaires à ceux trouvés par le laboratoire de contrôle de la 
station d'épuration de S'anaa pendant les années 2006 et 2007. En revanche, ces valeurs sont nettement supérieures par rapport à celles trouvées par Kabbour (2007) dans la station d'épuration de la ville du Rabat (Maroc).

Les valeurs moyennes de Nitrate enregistrées à la sortie de la station de traitement des eaux usées oscillent entre $12,73 \mathrm{mg} / \mathrm{L}$ et $17,73 \mathrm{mg} / \mathrm{L}$ comme valeurs extrêmes minimales et maximales avec une moyenne de $14,84 \mathrm{mg} / \mathrm{l}$. Ces valeurs se situent dans les limites autorisées selon l'OMS (2006).

\section{Rendements épuratoires}

Elimination de la charge organique $\left(\mathrm{DBO}_{5}\right.$, DCO Et MES)

Le Tableau 3, présente respectivement les rendements épuratoires en $\mathrm{MES}, \mathrm{DBO}_{5}$, DCO de l'effluent brut à l'entrée et de l'effluent épuré à la sortie de la STEP. Les rendements moyens d'abattement de la pollution sur la période de suivi sont de $86,4 \%$ pour les MES, 90,99\% pour la $\mathrm{DBO}_{5}$ et $89,99 \%$ (les performances épuratoires ont été supérieures à $85 \%(\mathrm{n}=24)$.) pour la DCO. Par contre, pour les MES, on observe de grandes fluctuations pour l'abattement global à la sortie de la STEP. On relève une variation importante des teneurs en MES avec un coefficient de variation de $23,32 \%$ et la teneur moyenne est de $110,6 \mathrm{mg} / \mathrm{l}$ avec un minimum observé de $81,43 \mathrm{mg} / \mathrm{l}$ et un maximum de 142,29 mg/l (Figure 3).

Les fortes valeurs en MES de l'effluent traité ont été observées surtout en mois de mai et de juin (cela est dû probablement au phénomène de dilation par les pluviales).

Les valeurs en DBO5 de l'effluent traité varient entre 91 et $111,5 \mathrm{mg} / \mathrm{l}$, à comparer au seuil maximum de $150 \mathrm{mg} / \mathrm{l}$ suggéré par la réglementation des normes du Yémen (ANRE, 1999a).

La qualité de l'effluent à la sortie, en terme de MES, répond fréquemment aux normes de rejets recommandées par la directive de L'Agence Nationale de
Ressource en Eau «< 1100 mg/L» (ANRE, 1999b).

\section{Elimination des sels (Conductivité)}

L'élimination des sels, dans les effluents de la STEP, a été analysée sous forme de la conductivité électrique exprimée en $\mu \mathrm{s} / \mathrm{cm}$ à $25^{\circ} \mathrm{C}$. La Figure 4 , présente la concentration de ce paramètre (moyennes mensuelles) mesurées dans l'effluent brut et traité de la STEP durant la période de l'étude. L'effluent traité présente en sortie des valeurs moyennes en conductivité de l'ordre de 2152,75 $\mu \mathrm{s} / \mathrm{cm}$ (Tableau 2 et Figure 4). Cette valeur moyenne est supérieure à la limite minimale fixée par la norme Yéménite (700$4000 \mu \mathrm{s} / \mathrm{cm}$ ) pour un rejet direct. D'après les résultats obtenus, l'élimination des sels est très faible. Les concentrations moyennes restent très élevées dans l'effluent traité et pourraient constituer un risque environnemental pour des rejets en milieu récepteurs.

\section{Rendement de nitrification et déphosphatation}

En phase d'anaérobiose, l'oxygène qui peut être apporté par les eaux usées ou par les retours de boue, réduit, voire inhibe le phénomène de relargage. Il en est de même pour les nitrates, car le sous-groupe des bactéries déphosphatantes aérobies facultatives (intervenant dans le processus de dénitrification) oxyde les acides gras à l'aide des nitrates (Deronzier et Choubert, 2002).

L'origine du phosphore dans les eaux usées est déduite de la connaissance des sources de phosphore naturel et de son utilisation; elle est multiple (Villebrun, 1989). Le phosphore provient : du métabolisme humain : un homme excrète entre 1 et 2 grammes de phosphore par jour. Il s'agit de l'apport principal en phosphore dans les cours d'eau (Dernat et al., 1994) ; des produits lessiviels et de nettoyage : 1 à 2 grammes de phosphore par jour et par habitation (en diminution), des rejets industriels : les effluents d'industries agroalimentaires, d'abattoirs, de laveries 
industrielles, d'industries de traitement de surface et d'industries chimiques spécialisées. Dans les régions relativement urbanisées, ils véhiculent une quantité de composés phosphorés à peu près équivalente à celle des eaux usées domestiques; les rejets agricoles ou d'origine naturelle sont retenus dans les sols et ne se retrouvent pas dans les eaux usées.

Le rendement d'élimination du phosphore dépend de différents facteurs. On peut citer la nature du réactif employé, le rapport molaire, le $\mathrm{pH}$ des boues, et la concentration initiale en phosphore. Le rendement d'élimination du phosphore est fortement dépendant de la concentration en phosphore des eaux usées comme en témoigne la Figure 5, exprimant le rendement d'élimination du phosphore. La composition de l'eau usée influence de manière prépondérante l'efficacité du processus de déphosphatation biologique. Cette influence est pratiquement aussi importante que la présence ou l'absence d'oxygène dans le milieu.

\section{Conclusion}

Cette étude a permis de suivre les performances d'une station d'épuration des eaux usées de $S$ 'anaa. Les résultats présentent des rendements épuratoires satisfaisants pour l'élimination des matières en suspension en accord avec les normes de rejet des eaux usées recommandées par la norme Yéménite pour ce paramètre $(<1100 \mathrm{mg} / \mathrm{l})$.

Dans $90,99 \%$ des mesures de la $\mathrm{DBO}_{5}$ sur l'effluent traité, les performances épuratoires ont été supérieures à $85 \%(\mathrm{n}=$ 24).

L'élimination des sels est très faible. Les concentrations moyennes restent très élevées dans l'effluent traité et pourraient constituer un risque environnemental pour des rejets en milieu récepteurs.

Cependant, ils constitueront un apport intéressant en sels dans le cas d'une réutilisation en agriculture selon les normes yéménites fixées pour les eaux usées destinées à l'irrigation.

\section{REFERENCES}

ANRE (Agence Nationale de Ressource en Eau) 1999a. Caractéristiques standard pour l'eau au Yémen, les eaux résiduaires industrielles et commerciales, L'Agence Nationale de Ressource en Eau, 6p.

ANRE (Agence Nationale de Ressource en Eau) 1999b. Caractéristiques standard pour l'eau au Yémen, les eaux utilisées dans l'irrigation, L'Agence Nationale de Ressource en Eau, 8p.

Arcand Y, Paris J, Rouleau D et Mayer R. 1989. Effet de paramètres d'opération sur la décantation de boues biologiques d'une unité de traitement d'eaux usées de raffinerie. Revue des Sciences de l'Eau, 2: 43 - 69.

Dernat M, Elmerich P, Pouillot M. 1994. Vers une optimisation de la déphosphatation physicochimique, L'Eau, l'Industrie, les Nuisances 182.

Deronzier G, Choubert J. 2002. Traitement $\mathrm{du}$ phosphore dans les petites stations d'épuration à boues activées. Comparaisons techniques et économiques des voies de traitement biologique et physico-chimique. Ministère de l'Agriculture, de l'Alimentation, de la Pêche et des Affaires rurales FNDAE $n^{\circ} 29$. Document technique.

El Guamri Y, Belghyti D. 2006. Etude de la qualité physico-chimique des eaux usées brutes de la commune urbaine de Saknia, rejetées dans le lac Fouarat (Kénitra, Maroc)". Journal Africain des Sciences de l'Environnement, 1 : 53-60.

IEA 2006. Institut local des eaux et d'assainissement, direction de la station d'épuration de la capitale Sana'a. Yémen.

Kabbour R. 2007: Appréciation des performances épuratrices du traitement par boues activées à faible et moyenne charge à la STEP pilote de Bouregreg (ONEP), Mémoire de $3^{\text {éme }}$ cycle, Fac, Sci, Kénitra, 76p. 
Maiga AH, Konate Y, Wethe J, Denyigba K, Zoungrana D, Togola L. 2006. Performances épuratoires d'une filière de trois étages de bassins de lagunage à microphytes sous climat sahélien : cas de la station de traitement des eaux usées de l'EIER. Sud Sciences \& Technologies, $14: 1-9$.

Metcalf Et Eddy, Inc. Wastewater engineering 1991. Treatment, Disposal and Reuse. 3ème Edition Library of Congress Cataloguing in Publication Data. TD. 645p. T34 (1991).

MPCI 2005. Impact environnemental de l'usage des eaux usées d'assainissement dans l'irrigation des agricultures, ministère de la planification et de la coopération internationale, juillet 2005.

NWSSIP, 2005. National Water Sector Strategy and Investment Program, 20052009, Republic of Yemen- Ministry of Water and Environment, Sana'a 2005, $67 \mathrm{p}$.
OMS 2006. A compendium of standards for wastewater reuse in the Eastern Moditerranean Region "Regional Office for the Eastern Moditerranean, Regional Centre for Environmental Health Activities (CEHA), 2006, WHOEM/CEH/142/E, 19p.

Raweh SMS. 2005. Pollution physicochimique et parasitologique $\mathrm{du}$ bas Sebou par les eaux usées urbaines de la ville de Kénitra (Maroc). Mémoire de $3^{\text {éme }}$ cycle, Fac, Sci, Kénitra, 78p.

Villebrun JF. 1989. La déphosphatation biologique appliquée à la station d'épuration de Craon, Rapport de la DDAF de la Mayenne. 